$11-1-2008$

\title{
Delete and Revise Procedures for Two-Stage Short- Run Control Charts
}

Matthew E. Elam

Texas Aઐ M University-Commerce, Matthew_Elam@tamu-commerce.edu

Follow this and additional works at: http://digitalcommons.wayne.edu/jmasm

Part of the Applied Statistics Commons, Social and Behavioral Sciences Commons, and the Statistical Theory Commons

\section{Recommended Citation}

Elam, Matthew E. (2008) "Delete and Revise Procedures for Two-Stage Short-Run Control Charts," Journal of Modern Applied Statistical Methods: Vol. 7 : Iss. 2 , Article 20.

DOI: $10.22237 /$ jmasm/1225513140

Available at: http://digitalcommons.wayne.edu/jmasm/vol7/iss2/20 


\title{
Delete and Revise Procedures for Two-Stage Short-Run Control Charts
}

\author{
Matthew E. Elam \\ Texas A\&M University-Commerce
}

This article investigates the effect different delete and revise procedures have on the performance of twostage short-run control charting methodology in the second stage of its two stage procedure. Five variables control chart combinations, six delete and revise procedures, and various out-of-control situations in both stages are considered.

Key words: Delete and revise, two-stage, short-run, control chart, probability of detection, run length, false alarm, computer program, FORTRAN.

\section{Introduction}

Control charting in short-run situations has received much attention in the literature. In a short-run situation, little or no historical information is available about a process in order to estimate process parameters to begin control charting. The application of two-stage control charting, which is used to determine the initial state of the process and the control limits for testing the future performance of the process, to short-run situations has resulted in a Shewhartbased control chart methodology with control chart factors for finite numbers of subgroups (Hillier, 1969; Yang \& Hillier, 1970).

The recent extension of this methodology to $(\overline{\mathrm{X}}, \mathrm{s})$ (Elam \& Case, 2005a) and (X, MR) (Elam \& Case, 2008) control charts, as well as the computerization of the control chart factor calculations for two-stage short run $(\bar{X}, \mathrm{R})$ (Elam \& Case, 2001), ( $\overline{\mathrm{X}}, \mathrm{v})$ and $(\bar{X}, \sqrt{v})$ (Elam \& Case, 2003), $(\bar{X}, s)$ (Elam \& Case, 2005b), and (X, MR) (Elam \& Case, 2006) has allowed for its further examination. Of particular interest is the effect that different delete and revise (D\&R) procedures have on the performance of the

Matthew E. Elam is an Associate Professor of Industrial Engineering and is an ASQ Certified Quality Engineer. Email: Matthew_Elam@tamu-commerce.edu. methodology in the second stage of the twostage procedure. A D\&R procedure removes out-of-control subgroups in stage one, allowing the data used to construct stage two control limits to be considered in-control. The removal of data in stage one becomes a more serious issue in a short-run situation because the less data available to construct stage two control limits, the less reliable they will be.

This article considers six different D\&R procedures for establishing control of a process in the first stage of the two-stage procedure. The first $D \& R$ procedure (D\&R 1) is from Hillier (1969), Ryan (1989), \& Montgomery (1997). It executes as follows:

i. Deletes out-of-control (OOC) initial subgroups on either the control chart for centering or spread entirely (i.e., if a subgroup shows OOC on either control chart, it is deleted from both charts).

ii. Recalculates control limits for both charts using the subgroups remaining after step $i$.

iii. Determines OOC subgroups.

iv. Repeats steps i-iii until no initial subgroups show OOC on either chart.

The second $D \& R$ procedure ( $D \& R 2)$ is from Pyzdek (1993). It executes as follows:

i. Deletes OOC initial subgroups on the control chart for spread.

ii. Recalculates control limits for the control chart for spread using the subgroups remaining after step $i$.

iii. Determines OOC subgroups.

iv. Repeats steps i-iii until no initial subgroups show OOC on the control chart for spread. 
v. Determines control limits for the chart for centering using the parameter estimate for spread obtained after completing steps i-iv and the overall average obtained from all of the initial subgroups.

vi. Repeats steps i-ii for the control chart for centering until no initial subgroups show OOC.

The third D\&R procedure ( $D \& R$ 3) is from Case (1998). It deletes OOC initial subgroups on the control chart for spread just once. No D\&R is performed on the control chart for centering.

The fourth D\&R procedure (D\&R 4) is from Doty (1997). It does not perform D\&R. This means that all initial subgroups are used to determine second stage control limits for both the control charts for centering and spread.

The fifth $D \& R$ procedure (D\&R 5) is a hybrid of D\&R 1 in that it iterates only once. It deletes OOC initial subgroups on either the control chart for centering or spread entirely (i.e., if a subgroup shows OOC on either control chart, it is deleted from both charts). D\&R is performed just once.

The sixth $D \& R$ procedure (D\&R 6) is a hybrid of D\&R 2 in that it iterates only once. It executes as follows:

i. Deletes OOC initial subgroups on the control chart for spread once.

ii. Determines the control limits for the chart for centering by using the parameter estimate for spread obtained after completing step $i$ and the overall average obtained from all initial subgroups.

iii. Performs step i for the control chart for centering.

Any of the six D\&R procedures may be used on two-stage short-run $(\overline{\mathrm{X}}, \mathrm{R}),(\overline{\mathrm{X}}, \mathrm{v})$, $(\overline{\mathrm{X}}, \sqrt{\mathrm{v}})$, and $(\overline{\mathrm{X}}, \mathrm{s})$ control charts. However, only D\&Rs 2, 3, 4, and 6 may be used on twostage short-run (X, MR) control charts because the MR values are calculated from two consecutive $\mathrm{X}$ values, thus no single MR value can be associated with a single $X$ value. Consequently, D\&Rs 1 and 5, which delete OOC initial subgroups on either the control chart for centering or spread entirely (i.e., if a subgroup shows OOC on either control chart, it is deleted from both charts), cannot be used on two-stage short-run (X, MR) control charts.

\section{Methodology}

The methodology for investigating the effect these six D\&R procedures have on the performance of two-stage short-run control charting in its second stage consists of three elements. The main element is the computer program that simulates two-stage short-run variables control charting. The second element, which is included in the operation of the program, is the measurements used to determine which D\&R procedure establishes the most reliable second stage control limits. The third element, which is explained using sample runs from the program, is the interpretation of the results from the program.

Measurements

The computer program presented here uses two sets of measurements to provide information that may be used to determine the reliability of second stage control limits. The first set of measurements is: the probability of detection (POD), the average run length (ARL), and the standard deviation of the run length (SDRL). The second set of measurements is: the probability of a false alarm ( $\mathrm{P}($ false alarm $))$, the average probability of a false alarm (APFL), and the standard deviation of the probability of a false alarm (SDPFL).

The POD is the probability that a control chart will signal, within a given number of subgroups following a shift, that a process is out-of-control (OOC). Additionally, if a process is in-control (IC), the POD may be interpreted as the probability of a Type I error (i.e., the probability of a false alarm) within a given number of subgroups starting with the first subgroup drawn from the process.

Using the POD allows for the characterization of the run length (RL) distribution. This is particularly useful in a short-run situation because it is desirable to know, for small numbers of subgroups, the probability of detecting a special cause signal or a false alarm. Using the ARL, which is the average number of subgroups that must be plotted on a control chart before an OOC 


\section{PROCEDURES FOR TWO-STAGE SHORT-RUN CONTROL CHARTS}

condition is indicated, in a short-run situation is not appropriate because a short-run may not last long enough to achieve the ARL. Additionally, as will be shown, the ARL can be misleading in choosing the appropriate D\&R procedure.

mathematically as:

The POD may be expressed

$$
\mathrm{POD}=\mathrm{P}(\mathrm{RL} \leq \mathrm{t})
$$

where $R L$ is the run length (in number of subgroups), $t$ is the subgroup number, and $\mathrm{P}(\mathrm{RL}$ $\leq \mathrm{t})$ is the probability that the RL is less than or equal to subgroup number $t$. As calculated by the computer program herein, for an OOC situation in the second stage of the two-stage procedure, the subgroup count starts at one at the first OOC subgroup. For an IC situation, the subgroup count starts at one with the first subgroup drawn from the process in the second stage.

Each time the program simulates twostage short-run variables control charting an RL value is determined. As the simulation is repeated, $R L$ and $R^{2}$ values are summed, and counts for the number of RLs less than or equal to each integer value in the interval $[1,50000]$ are kept. Once the repeating of the simulation is complete, the two sums are used to calculate the ARL and the SDRL, which is the standard deviation of the number of subgroups that must be plotted on a control chart before an OOC condition is indicated. The counts are used to determine the POD values.

For an OOC situation in the second stage of the two-stage procedure, it is desirable to have the highest possible POD values and the lowest possible ARL. For an IC situation in the second stage, it is desirable to have the lowest possible POD values and the highest possible ARL.

The probability of a false alarm ( $\mathrm{P}($ false alarm)) is the probability of a control chart indicating an OOC condition when none exists. Hillier's (1969) methodology, upon which the two-stage short-run variables control charts are based, allowed for the specification of the desired P(false alarm), that is, the desired Type I error probability.

The computer program presented here calculates the $\mathrm{P}$ (false alarm) when an OOC situation occurs beyond the first subgroup drawn from the process in the second stage of the two- stage procedure. Each time the program simulates two-stage short-run variables control charting under these conditions, a value for $\mathrm{P}$ (false alarm) is determined. As the simulation is repeated, $\mathrm{P}\left(\right.$ false alarm) and $\mathrm{P}(\text { false alarm })^{2}$ values are summed. Once the repeating of the simulation is complete, these two sums are used to calculate the APFL and the SDPFL. It is desirable for the $\mathrm{P}$ (false alarm) values, and consequently the APFL, to be as low as possible.

\section{The Computer Program}

The computer program that simulates two-stage short-run variables control charting is available starting at http://program.20m.com. It is coded in FORTRAN (1999). The program is intended to simulate two-stage short-run variables control charting of a process before initiating it so that a decision can be made regarding which $\mathrm{D} \& \mathrm{R}$ procedure to use when performing two-stage short-run variables control charting during the early run of the process. The $\mathrm{D} \& \mathrm{R}$ procedures provided by the program were described earlier; each segment of the program and its operation is now detailed.

The main program cc (control charting) includes the data entry, file setup, subroutine calls, summations of various values determined by the subroutines, final ARL, SDRL, P(false alarm), APFL, and SDPFL calculations, and the output of information to a file. It is the only segment of the program requiring user interaction.

The following inputs (in order of appearance in the program) are requested from the user in the main program cc:

- The process mean and standard deviation.

- The number of times to replicate the twostage short-run control charting procedure.

- The control chart combination: $(\overline{\mathrm{X}}, \mathrm{R})$, $(\bar{X}, v),(\bar{X}, \sqrt{v}),(\bar{X}, s)$, or $(X, M R)$.

- The subgroup size (not applicable to (X, MR) control charts).

- The number of subgroups for Stage 1.

- The choice of simulating the process in Stage 1 as IC or OOC. If OOC is chosen, the user is requested to enter the choice of a sustained shift in the mean, the standard deviation, or both. Once a shift type is selected, the program prompts for the shift 
size (in the same units as the parameter that has shifted) and the number of the first subgroup after the shift in Stage 1.

- The choice of simulating the process in Stage 2 as IC or OOC. If OOC is chosen, the user is requested to enter the choice of a sustained shift in the mean, the standard deviation, or both. Once the user chooses a shift type, the program prompts for the shift size (in the same units as the parameter that has shifted) and the number of the first subgroup after the shift in Stage 2.

- The choice of using a different starting value for seed for the Marse-Roberts Uniform ( 0 , 1) random variate generator (Marse \& Roberts, 1983) coded as subroutine random in module random_mod.

- The D\&R procedure (entered as 1, 2, 3, 4, 5, or 6). The program describes the execution of each D\&R procedure in detail for the user.

- The name (including the location) of the text file (extension .txt) containing the two-stage short-run control chart factors for the control chart combination entered earlier.

- The name (including the location) of the text file that will store the results from the program.

The second to last bullet point above requires further explanation. Appendix A shows the five input files that were used to generate the results in this study. The first input file contains the first and second stage short-run control chart factors for $(\bar{X}, \mathrm{R})$ charts from Table A4 in Elam $\&$ Case (2001) for $n=3$ and $m: 1-5$. The second input file contains the first and second stage short-run control chart factors for $(\overline{\mathrm{X}}, \mathrm{v})$ charts from Table A.4 in Elam \& Case (2003) for $n=3$ and $\mathrm{m}: 1-5$. The third input file contains the first and second stage short-run control chart factors for $(\bar{X}, \sqrt{\mathrm{v}})$ charts, also from Table A.4 in Elam $\&$ Case (2003) for $n=3$ and $m: 1-5$. The fourth input file contains the first and second stage short-run control chart factors for $(\overline{\mathrm{X}}, \mathrm{s})$ charts from Table A. 4 in Elam \& Case (2005b) for $n=3$ and $\mathrm{m}: 1-5$. The fifth input file contains the first and second stage short-run control chart factors for (X, MR) charts from Table 3 in Elam \& Case (2006) for m: 2-15.
The only difference between the appearance of the input files and their corresponding tables in their respective references is that the first stage short-run control chart factors in the first row of each input file are set to zero. This is required in order for the program to correctly read the second stage shortrun control chart factors from these input files when $\mathrm{m}=1$ (in the case of $(\overline{\mathrm{X}}, \mathrm{R}),(\overline{\mathrm{X}}, \mathrm{v})$, $(\bar{X}, \sqrt{v})$, and $(\bar{X}, s)$ control charts) or $\mathrm{m}=2$ (in the case of (X, MR) control charts).

When data entry is complete, the first replication of the two-stage short-run control charting procedure begins as program execution proceeds from main program $\mathrm{cc}$ to module Stage 1 and the subroutine for the control chart combination entered by the user. Each of the five subroutines for Stage 1 control charting performs the following tasks:

- Reads first stage short-run control chart factors from the input file.

- Generates first stage subgroups.

- Constructs first stage control limits.

- Determines OOC subgroups.

The tasks in the last two points use Hillier's (1969) approach. When Stage 1 control charting is complete, program execution returns to main program cc.

Once program execution returns to main program cc, it immediately proceeds to module D_and_R and the subroutine for the $D \& R$ procedure selected by the user. When the $\mathrm{D} \& \mathrm{R}$ procedure is complete, program execution returns to main program cc. At this point, the program assumes that control of the process has been established.

Once program execution returns to main program cc, required summations are calculated and required variable assignments are made. Program execution then proceeds to module Stage_2 and the subroutine for the control chart combination entered by the user. Each of the five subroutines for Stage 2 control charting performs the following tasks:

- Reads second stage short-run control chart factors from the input file.

- Constructs second stage control limits.

- Generates second stage subgroups. 


\section{PROCEDURES FOR TWO-STAGE SHORT-RUN CONTROL CHARTS}

- Determines the run length (RL) and, if applicable, if a false alarm occurs.

The calculations in the point above are based on the signaling capabilities of combined control charts for centering and spread; i.e., a signal occurs if a subgroup plots OOC on either the control chart for centering or the control chart for spread. The number of the first subgroup that signals is the RL value. The second stage control limits are not updated as subgroups are accumulated. When an RL value is determined, Stage 2 control charting is complete and program execution returns to main program cc.

In main program cc after Stage 2 control charting, required summations are calculated. When this is complete, execution returns to the location in main program cc immediately before the five subroutine calls for Stage 1 control charting to begin the second replication. The entire procedure for two-stage short-run control charting repeats for the number of times entered by the user.

After the last replication, program execution in main program $\mathrm{cc}$ proceeds to writing the following information to the output file:

- The process mean and standard deviation.

- The number of replications of the two-stage short-run control charting procedure that was carried out.

- The control chart combination $((\bar{X}, \mathrm{R})$, $(\overline{\mathrm{X}}, \mathrm{v}),(\overline{\mathrm{X}}, \sqrt{\mathrm{v}}),(\overline{\mathrm{X}}, \mathrm{s})$, or $(\mathrm{X}, \mathrm{MR}))$.

- The subgroup size (not applicable to (X, MR) control charts).

- The number of subgroups for Stage 1 .

- The D\&R procedure.

- The state of the process in Stage 1: IC or OOC. If it is OOC, then the type of sustained shift, the shift size (in the same units as the parameter that has shifted), and the number of the first subgroup after the shift in Stage 1 are given.

- The state of the process in Stage 2: IC or OOC. If it is OOC, then the type of sustained shift, the shift size (in the same units as the parameter that has shifted), and the number of the first subgroup after the shift in Stage 2 are given.

- The ARL and SDRL.
- The APFL and SDPFL (if applicable).

- A table of POD values.

The information in the first eight bullet points was entered by the user. The values in the last three bullet points are calculated by the program.

In addition to these calculated values, the computer program determines counts of the number of occurrences of certain events (when applicable). These events are as follows:

- The number of times out of the total number of replications that D\&R 1 iterated more than once.

- The number of times out of the total number of replications that D\&R 2 iterated more than once for the control chart for spread as well as for the control chart for centering.

- The number of times out of the total number of replications the program skipped a replication because the number of subgroups dropped to zero (for two-stage short-run $(\bar{X}, R),(\bar{X}, v),(\bar{X}, \sqrt{v}),(\bar{X}, s)$, and $(X$, $\mathrm{MR})$ control charts) or one (for two-stage short-run (X, MR) control charts) after OOC subgroups were deleted in a D\&R procedure.

- The number of times out of the total number of replications a D\&R procedure was stopped because the number of subgroups dropped to one (for two-stage short-run $(\bar{X}, \mathrm{R}),(\overline{\mathrm{X}}, \mathrm{v}),(\overline{\mathrm{X}}, \sqrt{\mathrm{v}})$, and $(\overline{\mathrm{X}}, \mathrm{s})$ control charts) or two (for two-stage short-run (X, MR) control charts) after OOC subgroups were deleted.

These counts, if applicable, are also written to the output file.

Once the above information, applicable calculations, and applicable counts have been written to the output file, execution of the computer program is complete.

\section{Results}

Fourteen pairs of tables (Tables 1a-14b) were constructed from output files generated from sample runs of the computer program. Tables $1 \mathrm{a}$ and $1 \mathrm{~b}$ are shown here. Tables $2 \mathrm{a}-14 \mathrm{~b}$ are available starting at http://program. $20 \mathrm{~m} . c 0 m$. For example, Tables $12 \mathrm{a}$ and $12 \mathrm{~b}$ were 
constructed using Sample Output File \#1 (see Appendix B) and Sample Output Files \#s 2-6 (available starting at http://program.20m.com). In addition to the notation already introduced in this article, Tables 1a-14b use the following notation:

- $\mathrm{MN}$ : a sustained shift in the mean

- SD: a sustained shift in the standard deviation

- MS: a sustained shift in both the mean and the standard deviation

- Replications (skipped): the number of replications carried out and, in parentheses, the number of replications skipped because the number of subgroups dropped to zero (for two-stage short-run $(\overline{\mathrm{X}}, \mathrm{R}),(\overline{\mathrm{X}}, \mathrm{v})$, $(\overline{\mathrm{X}}, \sqrt{\mathrm{v}}),(\overline{\mathrm{X}}, \mathrm{s})$, and $(\mathrm{X}, \mathrm{MR})$ control charts) or one (for two-stage short-run (X, MR) control charts) after OOC subgroups were deleted in a $D \& R$ procedure.

- Stops: the number of times out of the total number of replications carried out that a $D \& R$ procedure was stopped because the number of subgroups dropped to one (for two-stage short-run $(\overline{\mathrm{X}}, \mathrm{R}), \quad(\overline{\mathrm{X}}, \mathrm{v})$, $(\overline{\mathrm{X}}, \sqrt{\mathrm{v}})$, and $(\overline{\mathrm{X}}, \mathrm{s})$ control charts) or two (for two-stage short-run (X, MR) control charts) after OOC subgroups were deleted.

The sample runs of the program that generated the information in Tables 1a-14b assumed the following:

- The process mean and standard deviation are always 0.0 and 1.0 , respectively.

- The planned number of replications is always 5,000.

- The subgroup size $\mathrm{n}$ is always 3 (not applicable to (X, MR) control charts).

- The number of Stage 1 subgroups (denoted by $\mathrm{m}$ ) is always 5 for two-stage short-run $(\overline{\mathrm{X}}, \mathrm{R}),(\overline{\mathrm{X}}, \mathrm{v}),(\overline{\mathrm{X}}, \sqrt{\mathrm{v}})$, and $(\overline{\mathrm{X}}, \mathrm{s})$ control charts and it is always 15 for two-stage short-run (X, MR) control charts. This is why the first four sample input files (see Appendix A have two-stage short-run control chart factors for $(\bar{X}, R),(\bar{X}, v)$, $(\overline{\mathrm{X}}, \sqrt{\mathrm{v}})$, and $(\overline{\mathrm{X}}, \mathrm{s})$ charts for $\mathrm{m}$ up to and including $\mathrm{m}=5$ and the fifth sample input file (see Appendix A) has two-stage short-run control chart factors for (X, MR) charts for $\mathrm{m}$ up to and including $\mathrm{m}=15$.

- A shift in the mean is always of size 1.5 (same units as the mean).

- A shift in the standard deviation is always of size 1.0 (same units as the standard deviation).

- A shift in Stage 1 always occurs between subgroups 2 and 3 .

- A shift in Stage 2 always occurs between subgroups 10 and 11 .

- The process is IC immediately before Stage 2 control charting begins.

Sample Runs for an IC Process in Stages 1 and 2 The first 28 sample runs of the program are for the IC process during both Stage 1 and Stage 2 control charting. Two-stage short-run control charting for $(\overline{\mathrm{X}}, \mathrm{R}),(\overline{\mathrm{X}}, \mathrm{v}),(\overline{\mathrm{X}}, \sqrt{\mathrm{v}})$, $(\bar{X}, s)$, and $(X, M R)$ charts was simulated using all six D\&R procedures for each control chart combination. The results of these simulations appear in Tables 1a-5b.

Because the process is being simulated as IC in Stage 2, it is desirable for the ARL values in Tables 1a-5a to be as high as possible. Also, it is desirable for the $\mathrm{P}(\mathrm{RL} \leq \mathrm{t})$ values in Tables $1 b-5 b$ to be as low as possible (because they correspond to probabilities of false alarms within $t$ or less subgroups after starting Stage 2 control charting), especially for small numbers of subgroups (because a short-run situation is in effect).

Based on both of these criteria, the information in Tables $1 \mathrm{a}-5 \mathrm{~b}$ indicates that $\mathrm{D} \& \mathrm{R}$ 4 is, for the most part, the D\&R procedure of choice. The only exception is in Table $3 a$, where $D \& R \quad 1$ is the D\&R procedure of choice based on the ARL. This implies that, under the assumptions of this simulation, it is preferable to use subgroups that signal false alarms in the construction of second stage control limits. The cost, in terms of the loss in reliability of second stage control limits, is higher by throwing out subgroups that signal false alarms than it is by including them in the construction of second stage control limits. 
Table 1a: ARL, SDRL, Replications, and Stops for Two-Stage Short-Run $(\overline{\mathrm{X}}, \mathrm{R})$ Control Charts with Stage 1: IC and Stage 2: IC

\begin{tabular}{|c|c|c|c|c|}
\hline $\begin{array}{c}\text { D\&R } \\
\text { Procedure }\end{array}$ & ARL & SDRL & $\begin{array}{c}\text { Replications } \\
\text { (Skipped) }\end{array}$ & Stops \\
\hline 1 & 552.89 & 701.12 & $5000(0)$ & 0 \\
\hline 2 & 550.10 & 702.51 & 4999 (1) & 1 \\
\hline 3 & 552.87 & 701.72 & $5000(0)$ & 0 \\
\hline 4 & 560.49 & 702.22 & $5000(-----)$ & ----- \\
\hline 5 & 552.08 & 700.49 & $5000(0)$ & 0 \\
\hline 6 & 552.03 & 700.61 & $5000(0)$ & 0 \\
\hline \multicolumn{5}{|c|}{$\begin{array}{l}\text { \# of Times D\&R } 1 \text { Iterated More Than Once: } 22 \\
\text { \# of Times D\&R } 2 \text { Iterated More Than Once for the R Control Chart: } 8\end{array}$} \\
\hline
\end{tabular}

Table 1b: $\mathrm{P}(\mathrm{RL} \leq \mathrm{t})$ for Two-Stage Short-Run

$(\bar{X}, \mathrm{R})$ Control Charts with Stage 1: IC and Stage 2: IC

\begin{tabular}{|c|c|c|c|c|c|c|}
\hline \multirow{2}{*}{$\mathbf{t}$} & \multicolumn{7}{|c}{ Delete and Revise (D\&R) Procedure } \\
\cline { 2 - 7 } & $\mathbf{1}$ & $\mathbf{2}$ & $\mathbf{3}$ & $\mathbf{4}$ & $\mathbf{5}$ & $\mathbf{6}$ \\
\hline $\mathbf{1}$ & 0.00940 & 0.01000 & 0.00900 & 0.00740 & 0.00820 & 0.00860 \\
\hline $\mathbf{2}$ & 0.01640 & 0.01760 & 0.01600 & 0.01260 & 0.01520 & 0.01560 \\
\hline $\mathbf{3}$ & 0.02540 & 0.02741 & 0.02520 & 0.02040 & 0.02440 & 0.02500 \\
\hline $\mathbf{4}$ & 0.03360 & 0.03561 & 0.03300 & 0.02700 & 0.03260 & 0.03300 \\
\hline $\mathbf{5}$ & 0.03820 & 0.04061 & 0.03760 & 0.03140 & 0.03700 & 0.03760 \\
\hline $\mathbf{6}$ & 0.04400 & 0.04721 & 0.04400 & 0.03580 & 0.04320 & 0.04420 \\
\hline $\mathbf{8}$ & 0.05380 & 0.05761 & 0.05460 & 0.04520 & 0.05320 & 0.05480 \\
\hline $\mathbf{1 0}$ & 0.06400 & 0.06721 & 0.06480 & 0.05420 & 0.06380 & 0.06500 \\
\hline $\mathbf{1 5}$ & 0.08880 & 0.09182 & 0.08880 & 0.07820 & 0.08840 & 0.08920 \\
\hline $\mathbf{2 0}$ & 0.11040 & 0.11462 & 0.11100 & 0.09960 & 0.11000 & 0.11180 \\
\hline $\mathbf{3 0}$ & 0.14040 & 0.14423 & 0.14100 & 0.12980 & 0.13960 & 0.14180 \\
\hline $\mathbf{4 0}$ & 0.16480 & 0.16863 & 0.16520 & 0.15360 & 0.16420 & 0.16620 \\
\hline $\mathbf{5 0}$ & 0.19180 & 0.19584 & 0.19160 & 0.17980 & 0.19120 & 0.19320 \\
\hline $\mathbf{1 0 0}$ & 0.27440 & 0.27806 & 0.27460 & 0.26480 & 0.27440 & 0.27520 \\
\hline $\mathbf{2 0 0}$ & 0.40740 & 0.41148 & 0.40800 & 0.40060 & 0.40820 & 0.40820 \\
\hline $\mathbf{3 0 0}$ & 0.50200 & 0.50630 & 0.50340 & 0.49600 & 0.50360 & 0.50380 \\
\hline $\mathbf{4 0 0}$ & 0.57760 & 0.58192 & 0.57900 & 0.57320 & 0.57900 & 0.57940 \\
\hline $\mathbf{5 0 0}$ & 0.63500 & 0.63773 & 0.63640 & 0.63120 & 0.63600 & 0.63680 \\
\hline $\mathbf{7 5 0}$ & 0.74900 & 0.75075 & 0.74840 & 0.74560 & 0.74920 & 0.74860 \\
\hline $\mathbf{1 0 0 0}$ & 0.82100 & 0.82156 & 0.82060 & 0.81840 & 0.82120 & 0.82080 \\
\hline $\mathbf{2 0 0 0}$ & 0.95460 & 0.95479 & 0.95460 & 0.95280 & 0.95460 & 0.95480 \\
\hline $\mathbf{3 0 0 0}$ & 0.98480 & 0.98480 & 0.98480 & 0.98440 & 0.98500 & 0.98500 \\
\hline $\mathbf{5 0 0 0}$ & 0.99840 & 0.99840 & 0.99840 & 0.99860 & 0.99840 & 0.99840 \\
\hline $\mathbf{7 5 0 0}$ & 1.00000 & 1.00000 & 1.00000 & 1.00000 & 1.00000 & 1.00000 \\
\hline & & & & & & \\
\hline
\end{tabular}


Comparing results in Tables 1a-5a reveals that two-stage short-run $(\bar{X}, s)$ control charts have the highest ARL for D\&R 4. Comparing results in Tables $1 \mathrm{~b}-5 \mathrm{~b}$ reveals that two-stage short-run $(\overline{\mathrm{X}}, \sqrt{\mathrm{v}})$ control charts have, for most of the shown values of $t$, the lowest $P(R L \leq t)$ values for $D \& R 4$. These results imply that, under the assumptions of this simulation, different control chart combinations are preferable depending on the measurement used.

The information in Tables $1 b-4 b$ also indicates that the $P(R L \leq t)$ values when $t=1$ are reasonably close to the theoretical probability of a false alarm. Assuming independence between the control charts for centering and spread, the theoretical $\mathrm{P}$ (false alarm) may be calculated by:

$$
\begin{aligned}
& P(\text { false alarm })=\alpha_{C e n}+\left(\alpha_{\text {SpreadUCL }}+\right. \\
& \left.\alpha_{\text {Spread } L C L}\right)-\alpha_{C e n} \times\left(\alpha_{\text {SpreadUCL }}+\alpha_{\text {SpreadLCL }}\right)
\end{aligned}
$$

where $\alpha_{\text {Cen }}$ is the $\mathrm{P}$ (false alarm) on the control chart for centering, $\alpha_{\text {SpreadUCL }}$ is the $\mathrm{P}$ (false alarm) on the control chart for spread above the upper control limit (UCL), and $\alpha_{\text {SpreadLCL }}$ is the $\mathrm{P}$ (false alarm) on the control chart for spread below the lower control limit (LCL). For the sample runs of the program, $\alpha_{\text {Cen }}=0.0027$, $\alpha_{\text {SpreadUCL }}=0.005$, and $\alpha_{\text {SpreadLCL }}=0.001$. This means that $\mathrm{P}$ (false alarm), as calculated by equation (2), is equal to 0.0086838 .

For example, the $\mathrm{P}(\mathrm{RL} \leq \mathrm{t})$ value from Table $1 b$ for D\&R 1 and $t=1$ is 0.00940 . The fact that this value is reasonably close to the theoretical probability of a false alarm is not surprising. As mentioned previously, Hillier's (1969) methodology, upon which the two-stage short-run variables control charts are based, allowed for the specification of the desired probability of a false alarm.

In Table $5 \mathrm{~b}$, each of the $\mathrm{P}(\mathrm{RL} \leq \mathrm{t})$ values for $\mathrm{t}=1$ are much lower than 0.0086838 . The closest one is $60.847 \%$ smaller than 0.0086838 . However, these lower $\mathrm{P}(\mathrm{RL} \leq \mathrm{t})$ values for $\mathrm{t}=1$ come at the price of having the lowest ARL for
D\&R 4 among Tables 1a-5a. This is an example of the tradeoff mentioned by Del Castillo (1995) between having a low probability of a false alarm and a high probability of detecting a special cause signal inherent with two-stage short-run control charts.

The information in Tables 1a-5a also indicates that $D \& R \quad 1$ and $D \& R \quad 2$ are iterating more than once. These multiple iterations seem to create conditions causing replications to be skipped and the chosen D\&R procedure to be stopped. Also, if confidence intervals were constructed using the ARL and SDRL values in Tables 1a-5a, then, depending on the confidence level chosen, the ARL results in Tables 1a-5a may not be statistically significantly different.

Sample Runs for an OOC Process in Stage 1 and an IC Process in Stage 2

The next 18 sample runs of the program were for the process being OOC during Stage 1 control charting and IC during Stage 2 control charting. Two-stage short-run control charting for $(\bar{X}, R)$ charts was simulated using all six D\&R procedures for each OOC condition (MN, $\mathrm{SD}, \mathrm{MS})$. The results of these simulations are shown in Tables 6a-8b.

Because the process is being simulated as IC in Stage 2, it is desirable for the ARL values in Tables $6 a-8 \mathrm{a}$ to be as high as possible. Also, it is desirable for the $\mathrm{P}(\mathrm{RL} \leq \mathrm{t})$ values in Tables $6 \mathrm{~b}-8 \mathrm{~b}$ to be as low as possible (because they correspond to probabilities of false alarms within $t$ or less subgroups after starting Stage 2 control charting), especially for small numbers of subgroups (since a short-run situation is in effect).

Based on the ARL, Tables $6 \mathrm{a}-8 \mathrm{a}$ indicate that $D \& R \quad 1$ was the procedure of choice, regardless of the OOC condition in Stage 1. However, the SDRL values for D\&R 1 are higher than those for the other D\&R procedures. The ARL for D\&R 1 in Table $7 \mathrm{a}$ is higher than the ARL values for D\&R 1 in Tables $6 \mathrm{a}$ and $8 \mathrm{a}$. The ARL for D\&R 1 in Table $6 \mathrm{a}$ is the lowest of the three. These results imply that, under the assumptions of this simulation, the type of OOC condition in Stage 1 has an effect on the IC ARL in Stage 2. Additionally, the ARL values for each of the six D\&R procedures in Table la are 


\section{PROCEDURES FOR TWO-STAGE SHORT-RUN CONTROL CHARTS}

higher than the respective ARL values in Tables 6a-8a. This result implies that, under the assumptions of this simulation, an OOC condition in Stage 1 caused a reduction in the IC ARL in Stage 2, regardless of the D\&R procedure used.

The choice of the appropriate $\mathrm{D} \& \mathrm{R}$ procedure based on the $\mathrm{P}(\mathrm{RL} \leq \mathrm{t})$ values in Tables $6 \mathrm{~b}-8 \mathrm{~b}$ varies depending on the OOC condition as well as the subgroup number t. In Table $6 b$, $D \& R \quad 4$ results in the lowest $P(R L \leq t)$ values for shown values of $t \leq 10$. For shown values of $t>$ $10, D \& R \quad 1$ is the D\&R procedure of choice. In Table $7 b, D \& R \quad 4$ again results in the lowest $P(R L \leq t)$ values, but for shown values of $t \leq 300$. For most of the shown values of $t \geq 300, D \& R 1$ is the $\mathrm{D} \& \mathrm{R}$ procedure of choice. In Table $8 \mathrm{~b}$, $D \& R \quad 1$ results in the lowest $P(R \leq t)$ values for each of the shown values of $t$ except $t$ : 30,40 , 50. Because D\&R 1 is not the procedure of choice in Tables $6 \mathrm{~b}$ and $7 \mathrm{~b}$ for shown values of $t$ $\leq 10$ and $\mathrm{t} \leq 200$, respectively, this is an example of how the ARL can be misleading in choosing the appropriate $\mathrm{D} \& \mathrm{R}$ procedure to use in a shortrun situation.

The results from Tables $6 \mathrm{~b}$ and $7 \mathrm{~b}$ imply that, under the assumptions of this simulation, it is preferable to use subgroups that signal shifts in either the mean or the standard deviation in the construction of second stage control limits. The cost, in terms of the loss in reliability of second stage control limits, is higher by throwing out subgroups that signal shifts in either the mean or the standard deviation than it is by including them in the construction of second stage control limits.

The $\mathrm{P}(\mathrm{RL} \leq \mathrm{t})$ values for shown values of $t \leq 300$ for D\&R 4 and for shown values of $t \geq$ 300 for D\&R 1 in Table $7 \mathrm{~b}$ are lower than the lowest $P(R L \leq t)$ values in Tables $6 \mathrm{~b}$ and $8 \mathrm{~b}$. The lowest $P(R L \leq t)$ values in Table $6 b$ are higher than those in Tables $7 \mathrm{~b}$ and $8 \mathrm{~b}$. These results imply that, under the assumptions of this simulation, the type of OOC condition in Stage 1 has an effect on the $P(R L \leq t)$ values in Stage 2 . Additionally, the lowest $\mathrm{P}(\mathrm{RL} \leq \mathrm{t})$ values in Table $1 \mathrm{~b}$ are higher than those in Table $7 \mathrm{~b}$ for shown values of $\mathrm{t} \leq 200$ and in Table $8 \mathrm{~b}$ for shown values of $t \leq 100$. These results imply that, under the assumptions of this simulation, having Stage
1 IC does not necessarily result in Stage 2 control limits with the lowest $\mathrm{P}(\mathrm{RL} \leq \mathrm{t})$ values.

An issue of concern is the $\mathrm{P}(\mathrm{RL} \leq \mathrm{t})$ values when $\mathrm{t}=1$. In Table $6 \mathrm{~b}$, each of these values is much larger than 0.0086838 , the theoretical probability of a false alarm. The closest one is $396.140 \%$ larger than 0.0086838 . In Table $7 \mathrm{~b}$, each of these values is much smaller than 0.0086838 . The closest one is $241.217 \%$ smaller than 0.0086838 . In Table 8 b, some of these values are reasonably close to 0.0086838 , although others are not. These results are in contrast to the $\mathrm{P}(\mathrm{RL} \leq \mathrm{t})$ values when $t=1$ in Table $1 b$. Clearly, under the assumptions of this simulation, an OOC condition as well as the type of OOC condition in Stage 1 has a significant effect on the $\mathrm{P}(\mathrm{RL} \leq \mathrm{t})$ values when $\mathrm{t}=1$ in Stage 2 .

Again, the information in Tables 6a-8a indicates that D\&R 1 and D\&R 2 are iterating more than once. These multiple iterations seem to create conditions causing replications to be skipped and the chosen D\&R procedure to be stopped. Also, if confidence intervals were constructed using the ARL and SDRL values in Tables $6 \mathrm{a}-8 \mathrm{a}$, then, depending on the confidence level chosen, the ARL results in Tables 6a-8a may not be statistically significantly different.

Sample Runs for an IC Process in Stage 1 and an OOC Process in Stage 2

The next 18 sample runs of the program were for the process being IC during Stage 1 control charting and OOC during Stage 2 control charting. Two-stage short-run control charting for $(\bar{X}, \mathrm{R})$ charts was simulated using all six D\&R procedures for each OOC condition (MN, $\mathrm{SD}, \mathrm{MS})$. The results of these simulations are shown in Tables 9a-11b.

Because the process is being simulated as OOC in Stage 2, it is desirable for the ARL and the APFL values in Tables 9a-11a to be as low as possible. Also, it is desirable for the $\mathrm{P}(\mathrm{RL} \leq \mathrm{t})$ values in Tables $9 \mathrm{~b}-11 \mathrm{~b}$ to be as high as possible (because they correspond to probabilities of detecting special causes within $\mathrm{t}$ or less subgroups after the shift in Stage 2), especially for small numbers of subgroups (because a short-run situation is in effect). 
Based on the ARL, D\&R 2 (in Tables 9a and 11a) and D\&R 4 (in Table 10a) are the D\&R procedures of choice. The ARL for D\&R 2 in Table 11a is lower than the ARL values for D\&Rs 2 and 4 in Tables $9 \mathrm{a}$ and 10a, respectively. The ARL for D\&R 2 in Table 9a is the highest of the three (it is $1423.680 \%$ larger than the ARL for D\&R 2 in Table 11a). These results imply that, under the assumptions of this simulation, the type of OOC condition in Stage 2 has an effect on the OOC ARL in Stage 2. As expected, the ARL values for each of the six D\&R procedures in Tables 9a-11a are much lower than the respective ARL values in Table $1 \mathrm{a}$.

Based on the APFL, Tables 9a-11a indicate that $D \& R \quad 4$ is the $D \& R$ procedure of choice regardless of the OOC condition in Stage 2. This reaffirms the statement that, in terms of the APFL, it is preferable to use subgroups that signal false alarms in the construction of second stage control limits. Also, the APFL values for D\&R 4 are reasonably close to 0.0086838 , the theoretical probability of a false alarm. However, the APFL values for the other D\&R procedures are slightly inflated.

The choice of the appropriate $\mathrm{D} \& \mathrm{R}$ procedure based on the $\mathrm{P}(\mathrm{RL} \leq \mathrm{t})$ values varies depending on the OOC condition as well as the subgroup number t. In Table 9b, D\&R 2 results in the highest $\mathrm{P}(\mathrm{RL} \leq \mathrm{t})$ values for shown values of $t \leq 200$ (except $t=4$ ). In Table 10b, D\&Rs 5 (for shown values of $t \leq 10$ (except $t=1)$ ), 2 (for shown values of $t \geq 15$ and $t \leq 100$ ), and 4 (for shown values of $t \geq 200$ ) result in the highest $\mathrm{P}(\mathrm{RL} \leq \mathrm{t}$ ) values. In Table $11 \mathrm{~b}$, D\&Rs 2 (for shown values of $t \leq 200$, except $t=1$ ) and 4 (for shown values of $t \geq 100$ ) result in the highest $\mathrm{P}(\mathrm{RL} \leq \mathrm{t})$ values. Because the ARL value in Table 10a is not the lowest for D\&R 2 or D\&R 5 , this is another example of how the ARL can be misleading in choosing the appropriate $\mathrm{D} \& \mathrm{R}$ procedure in a short-run situation.

The largest $P(R L \leq t)$ values in Table $11 \mathrm{~b}$ are larger than the largest $\mathrm{P}(\mathrm{RL} \leq \mathrm{t})$ values in Tables $9 \mathrm{~b}$ and $10 \mathrm{~b}$. The largest $\mathrm{P}(\mathrm{RL} \leq \mathrm{t})$ values in Table $9 \mathrm{~b}$ are lower than those in Tables $10 \mathrm{~b}$ and $11 \mathrm{~b}$. These results imply that, under the assumptions of this simulation, the type of OOC condition in Stage 2 has an effect on the $P(R L \leq t)$ values in Stage 2. As expected, the $P(R L \leq t)$ values for each of the six $D \& R$ procedures in Tables 9b-11b are much higher than the respective $P(R L \leq t)$ values in Table $1 \mathrm{a}$.

The information in Tables 9a-11b presents another example of the tradeoff mentioned by Del Castillo (1995) between having a low probability of a false alarm and a high probability of detecting a special cause signal inherent with two-stage short-run control charts. Although D\&R 4 results in the lowest APFL values regardless of the OOC condition in Stage 2, it also results in the lowest $P(R L \leq t)$ values for many of the shown values of $t$ in Tables $9 \mathrm{~b}$ and $10 \mathrm{~b}$.

Again, the information in Tables 9a-11a indicates that D\&R 1 and D\&R 2 are iterating more than once. These multiple iterations seem to create conditions causing replications to be skipped and the chosen D\&R procedure to be stopped. Also, if confidence intervals were constructed using the ARL and SDRL values in Tables 9a-11a, then, depending on the confidence level chosen, the ARL results in Tables 9a-11a may not be statistically significantly different.

Sample Runs for an OOC Process in Stages 1 and 2

The final 18 sample runs of the program were for the process being OOC during both Stage 1 and Stage 2 control charting. Two-stage short-run control charting for $(\overline{\mathrm{X}}, \mathrm{R})$ charts was simulated using all six D\&R procedures for each OOC condition (MN, SD, MS) in Stage 1 and one OOC condition (MN) in Stage 2. The results of these simulations are shown in Tables 12a$14 b$.

Because the process was simulated as OOC in Stage 2, it is desirable for the ARL and the APFL values in Tables 12a-14a to be as low as possible. Also, it is desirable for the $P(R L \leq t)$ values in Tables $12 \mathrm{~b}-14 \mathrm{~b}$ to be as high as possible (because they correspond to probabilities of detecting special causes within $t$ or less subgroups after the shift in Stage 2), especially for small numbers of subgroups (because a short-run situation is in effect).

Based on the ARL, D\&R 2 (in Tables 12a and 14a) and D\&R 3 (in Table 13a) are the 


\section{PROCEDURES FOR TWO-STAGE SHORT-RUN CONTROL CHARTS}

D\&R procedures of choice. The ARL for D\&R 3 in Table 13a is lower than the ARL values for D\&R 2 in Tables 12a and 14a. The ARL for D\&R 2 in Table $14 \mathrm{a}$ is the highest of the three. These results imply that, under the assumptions of this simulation, the type of OOC condition in Stage 1 has an effect on the OOC (MN) ARL in Stage 2. Additionally, the ARL values for each of the six D\&R procedures in Table 9a are much lower than the respective ARL values in Tables 12a-14a. This implies that, under the assumptions of this simulation, an OOC condition in Stage 1 causes an increase in the OOC (MN) ARL in Stage 2, regardless of the $\mathrm{D} \& \mathrm{R}$ procedure used.

Based on the APFL, Tables 12a-14a indicate that D\&R 4 is the procedure of choice regardless of the OOC condition in Stage 1. This implies that, under the assumptions of this simulation, it is preferable to use subgroups that signal shifts in the mean, the standard deviation, or both in the construction of second stage control limits. The cost, in terms of the loss in reliability of second stage control limits, is higher by throwing out subgroups that signal shifts in the mean, the standard deviation, or both than it is by including them in the construction of second stage control limits. Additionally, comparing the APFL results in Table 9a with those in Tables 12a-14a reveals that, under the assumptions of this simulation, an MN in Stage 1 has the effect of increasing the APFL (see Table 12a) and an SD in Stage 1 has the effect of decreasing the APFL (see Table 13a).

An issue of concern is the differences in the APFL values from 0.0086838 , the theoretical probability of a false alarm. The APFL value for D\&R 4 in Table 12a is $369.424 \%$ larger than 0.0086838 . The APFL values for D\&R 4 in Tables $13 \mathrm{a}$ and $14 \mathrm{a}$ are $65.683 \%$ and $33.209 \%$, respectively, smaller than 0.0086838 . These results are somewhat consistent with those regarding the $\mathrm{P}(\mathrm{RL} \leq \mathrm{t})$ values when $\mathrm{t}=1$ in Tables $6 \mathrm{~b}-8 \mathrm{~b}$. Clearly, under the assumptions of this simulation, the type of OOC condition in Stage 1 has a significant effect on the APFL values before the shift in Stage 2.

Based on the $P(R L \leq t)$ values, $D \& R 2$ is the appropriate procedure for most of the shown values of $t$ regardless of the $\mathrm{OOC}$ condition in
Stage 1. Because Table 13a indicates that D\&R 3 is the D\&R procedure of choice, this is another example of how the ARL can be misleading in choosing the appropriate $\mathrm{D} \& \mathrm{R}$ procedure in a short-run situation. The fact that the largest $\mathrm{P}(\mathrm{RL} \leq \mathrm{t})$ values in Table $14 \mathrm{~b}$ are lower than those in Tables $12 b$ and $13 b$ for most of the shown values of $t$ implies that, under the assumptions of this simulation, the type of OOC condition in Stage 1 has an effect on the $\mathrm{P}(\mathrm{RL} \leq \mathrm{t})$ values in Stage 2.

Additionally, the largest $\mathrm{P}(\mathrm{RL} \leq \mathrm{t})$ values in Table $9 \mathrm{~b}$ are larger than those in Tables $12 \mathrm{~b}$ 14b. This result implies that, under the assumptions of this simulation, an OOC condition in Stage 1 decreases the $\mathrm{P}(\mathrm{RL} \leq \mathrm{t})$ values in Stage 2; this is not desirable due to the $\mathrm{MN}$ in Stage 2. However, it is desirable for Stage 2 IC as was the case in comparing results in Table $1 \mathrm{~b}$ to those in Tables 6b-8b. Clearly, under the assumptions of this simulation, when one is interested in detecting MN in Stage 2, it is highly desirable to have the process IC when drawing first stage subgroups.

The information in Tables 12a-14b presents another example of the tradeoff mentioned by Del Castillo (1995) between having a low probability of a false alarm and a high probability of detecting a special cause signal inherent with two-stage short-run control charts. Although D\&R 4 results in the lowest APFL values regardless of the OOC condition in Stage 1 , it also results in the lowest $P(R L \leq t)$ values for many of the shown values of $t$ in Tables $13 \mathrm{~b}$ and $14 \mathrm{~b}$.

Again, as in the three previous subsections, the information in Tables 12a-14a indicates that D\&R 1 and $D \& R 2$ are iterating more than once. These multiple iterations seem to create conditions causing replications to be skipped and the chosen D\&R procedure to be stopped. Also, if confidence intervals were constructed using the ARL and SDRL values in Tables 12a-14a, then, depending on the confidence level chosen, the ARL results in Tables 12a-14a may not be statistically significantly different. 


\section{Conclusion}

The interpretation of the sample runs of the computer program establish the fact that no hard and fast rules can be developed regarding which $\mathrm{D} \& \mathrm{R}$ procedure is appropriate when performing two-stage short-run variables control charting. Under the assumptions of the simulations performed, the choice of the appropriate D\&R procedure varies both among and within measurements, among control chart combinations, among IC and various OOC conditions in both stages, and among numbers of subgroups plotted in Stage 2. It may be possible that the choice of the appropriate $D \& R$ procedure varies among shift sizes and the timing of shifts, though this was not investigated.

If decisions cannot be made regarding values for these variables, then extensive sample runs similar to the ones in the previous section need to be performed. However, if certain values for these variables are desired, then the process of making sample runs and interpreting their results is much simpler.

\section{References}

Case, K. E. (1998). The delete and revise (D\&R) procedure. In Class Notes / Lecture Material. Stillwater, Oklahoma: Oklahoma State University.

Del Castillo, E. (1995). Discussion. Journal of Quality Technology, 27(4), 316-321.

Doty, L. A. (1997). SPC for short run manufacturing. Cincinnati, OH: Hanser Gardner Publications.

Elam, M. E. \& Case, K. E. (2001). A computer program to calculate two-stage shortrun control chart factors for $(\bar{X}, \mathrm{R})$ charts. Quality Engineering, 14(1), 77-102.
Elam, M. E. \& Case, K. E. (2005b). A computer program to calculate two-stage shortrun control chart factors for $(\overline{\mathrm{X}}, \mathrm{s})$ charts. Quality Engineering, 17(2), 259-277.

Elam, M. E. \& Case, K. E. (2006). A computer program to calculate two-stage shortrun control chart factors for (X, MR) charts. Journal of Statistical Software, 15(11), http://www.jstatsoft.org/.

Elam, M. E. \& Case, K. E. (2003). A computer program to calculate two-stage shortrun control chart factors for $(\overline{\mathrm{X}}, \mathrm{v})$ and $(\overline{\mathrm{X}}, \sqrt{\mathrm{v}})$ charts. Quality Engineering, 15(4), 609-638.

Elam, M. E. \& Case, K. E. (2005a). Two-stage short-run $(\overline{\mathrm{X}}, \mathrm{s})$ control charts. Quality Engineering, 17(1), 95-107.

Elam, M. E. \& Case, K. E. (2008). Twostage short-run (X, MR) control charts. Journal of Modern Applied Statistical Methods, 7(1), 275-285.

FORTRAN (1999). Essential Lahey FORTRAN 90 compiler release 4.00c. Incline Village, NV: Lahey Computer Systems, Inc.

Hillier, F. S. (1969). X - and R-chart control limits based on a small number of subgroups. Journal of Quality Technology, 1(1), $17-26$

Marse, K. \& Roberts, S. D. (1983). Implementing a portable FORTRAN uniform (0, 1) generator. Simulation, 41(4), 135-139.

Montgomery, D. C. (1997). Introduction to statistical quality control, $3^{\text {rd }}$ Ed. New York, NY: John Wiley and Sons, Inc.

Pyzdek, T. (1993). Process control for short and small runs. Quality Progress, 26(4), 51-60.

Ryan, T. P. (1989). Statistical methods for quality improvement. New York, NY: John Wiley and Sons, Inc.

Yang, C.-H. \& Hillier, F. S. (1970). Mean and variance control chart limits based on a small number of subgroups. Journal of Quality Technology, 2(1), 9-16. 


\section{PROCEDURES FOR TWO-STAGE SHORT-RUN CONTROL CHARTS}

\section{Appendix A}

Sample Input File Containing First and Second Stage Short Run Control Chart Factors for $(\bar{X}, R)$ Charts for $n=3$ and $m: 1-5$

$\begin{array}{llllll}0.00000 & 0.00000 & 0.00000 & 8.35221 & 14.34466 & 0.03152 \\ 1.56033 & 1.86966 & 0.06112 & 2.70257 & 5.65885 & 0.03337 \\ 1.35226 & 2.21659 & 0.04924 & 1.91239 & 4.27295 & 0.03407 \\ 1.25601 & 2.35005 & 0.04491 & 1.62151 & 3.74247 & 0.03443 \\ 1.20246 & 2.41685 & 0.04267 & 1.47271 & 3.46631 & 0.03465\end{array}$

Sample Input File Containing First and Second Stage Short Run Control Chart Factors for $(\overline{\mathrm{X}}, \mathrm{v})$ Charts for $\mathrm{n}=3$ and $\mathrm{m}: 1-5$

$\begin{array}{llllll}0.00000 & 0.00000 & 0.00000 & 17.69484 & 199.00000 & 0.00100100 \\ 2.87519 & 1.99000 & 0.00200000 & 4.97997 & 26.28427 & 0.00100075 \\ 2.40967 & 2.78787 & 0.00150038 & 3.40779 & 14.54411 & 0.00100067 \\ 2.20599 & 3.31601 & 0.00133378 & 2.84792 & 11.04241 & 0.00100063 \\ 2.09497 & 3.67043 & 0.00125047 & 2.56580 & 9.42700 & 0.00100060\end{array}$

Sample Input File Containing First and Second Stage Short Run Control Chart Factors for $(\bar{X}, \sqrt{v})$ Charts for $n=3$ and $m: 1-5$

$\begin{array}{llllll}0.00000 & 0.00000 & 0.00000 & 17.69484 & 15.91775 & 0.03570 \\ 2.87519 & 1.59177 & 0.05046 & 4.97997 & 5.45415 & 0.03365 \\ 2.40967 & 1.77629 & 0.04121 & 3.40779 & 3.97519 & 0.03297 \\ 2.20599 & 1.89811 & 0.03807 & 2.84792 & 3.42822 & 0.03263 \\ 2.09497 & 1.97649 & 0.03648 & 2.56580 & 3.14794 & 0.03243\end{array}$

Sample Input File Containing First and Second Stage Short Run Control Chart Factors for $(\bar{X}, s)$ Charts for $n=3$ and $m: 1-5$

$\begin{array}{llllll}0.00000 & 0.00000 & 0.00000 & 15.68165 & 14.10674 & 0.03164 \\ 2.95828 & 1.86761 & 0.06134 & 5.12390 & 5.60680 & 0.03348 \\ 2.57119 & 2.21123 & 0.04940 & 3.63621 & 4.24135 & 0.03417 \\ 2.39128 & 2.34285 & 0.04505 & 3.08713 & 3.71725 & 0.03453 \\ 2.29099 & 2.40840 & 0.04280 & 2.80588 & 3.44396 & 0.03476\end{array}$

Sample Input File Containing First and Second Stage Short Run Control Chart Factors for (X, MR) Charts for m: 2-15

$\begin{array}{llllll}0.00000 & 0.00000 & 0.00000 & 204.19466 & 127.32134 & 0.00157 \\ 22.24670 & 2.95360 & 0.00235 & 31.46159 & 26.11886 & 0.00157 \\ 10.72641 & 3.58790 & 0.00209 & 13.84773 & 13.20218 & 0.00157 \\ 7.34996 & 3.83736 & 0.00196 & 9.00182 & 9.27880 & 0.00157 \\ 5.87022 & 3.89898 & 0.00188 & 6.94574 & 7.52080 & 0.00157 \\ 5.06862 & 3.89368 & 0.00183 & 5.85274 & 6.55349 & 0.00157 \\ 4.57470 & 3.86822 & 0.00179 & 5.18723 & 5.95038 & 0.00157 \\ 4.24308 & 3.83885 & 0.00177 & 4.74391 & 5.54166 & 0.00157 \\ 4.00644 & 3.81088 & 0.00175 & 4.42928 & 5.24776 & 0.00157 \\ 3.82972 & 3.78583 & 0.00173 & 4.19525 & 5.02691 & 0.00157 \\ 3.69307 & 3.76385 & 0.00171 & 4.01479 & 4.85521 & 0.00157 \\ 3.58441 & 3.74470 & 0.00170 & 3.87161 & 4.71806 & 0.00157 \\ 3.49606 & 3.72800 & 0.00169 & 3.75537 & 4.60610 & 0.00157 \\ 3.42287 & 3.71338 & 0.00168 & 3.65920 & 4.51303 & 0.00157\end{array}$




\section{ELAM}

Appendix B: Sample Output File \#1

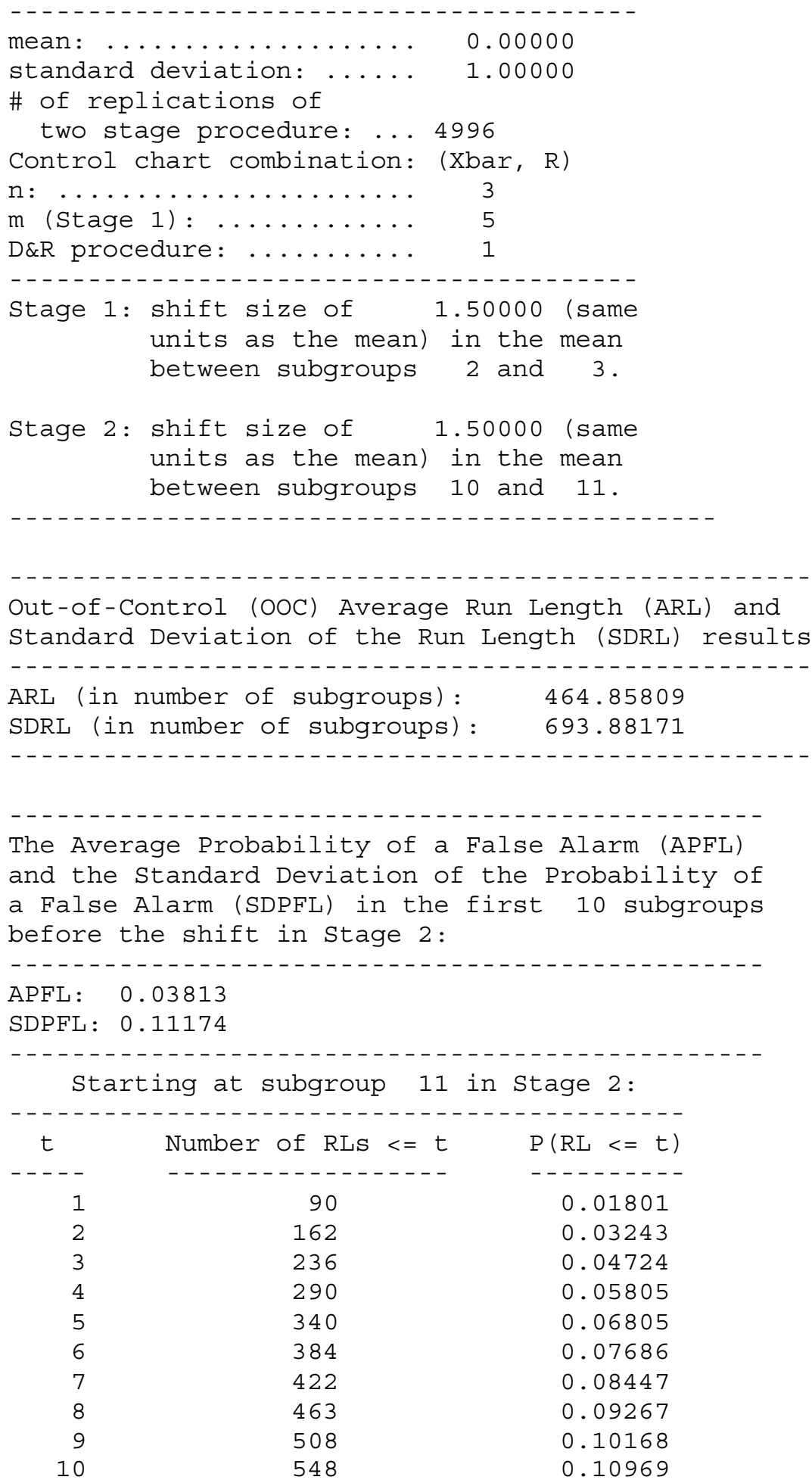


PROCEDURES FOR TWO-STAGE SHORT-RUN CONTROL CHARTS

Appendix B: Sample Output File \#1 (continued)

$\begin{array}{rrr}15 & 674 & 0.13491 \\ 20 & 793 & 0.15873 \\ 30 & 1002 & 0.20056 \\ 40 & 1162 & 0.23259 \\ 50 & 1277 & 0.25560 \\ 75 & 1550 & 0.31025 \\ 100 & 1781 & 0.35649 \\ 200 & 2432 & 0.48679 \\ 300 & 2893 & 0.57906 \\ 400 & 3259 & 0.65232 \\ 500 & 3504 & 0.70136 \\ 750 & 3997 & 0.80004 \\ 1000 & 4296 & 0.85989 \\ 2000 & 4814 & 0.96357 \\ 3000 & 4934 & 0.98759 \\ 4000 & 4973 & 0.99540 \\ 5000 & 4984 & 0.99760 \\ 7500 & 4994 & 0.99960 \\ 10000 & 4995 & 0.99980 \\ 20000 & 4996 & 1.00000 \\ 30000 & 4996 & 1.00000 \\ 40000 & 1.00000 \\ 50000 & 4996 & 1.00000 \\ -------------\end{array}$

The first D\&R procedure iterated more than once a total of 111 time (s).

Replications skipped 4 time(s) because the number of subgroups dropped to zero after out-of-control (OOC) subgroups were deleted.

D\&R procedure 1 stopped 12 time (s) because the number of subgroups dropped to one after out-of-control (OOC) subgroups were deleted. 